\title{
Distributed on-line multidimensional scaling for self-localization in wireless sensor networks
}

\author{
G. Morral ${ }^{\mathrm{a}, 1}$, P. Bianchi ${ }^{\mathrm{a}}$ \\ ${ }^{a}$ Institut Mines-Télécom, Télécom ParisTech, CNRS LTCI. 46, rue Barrault, 75013 Paris, France.
}

\begin{abstract}
The present work considers the localization problem in wireless sensor networks formed by fixed nodes. Each node seeks to estimate its own position based on noisy measurements of the relative distance to other nodes. In a centralized batch mode, positions can be retrieved (up to a rigid transformation) by applying Principal Component Analysis (PCA) on a so-called similarity matrix built from the relative distances. In this paper, we propose a distributed on-line algorithm allowing each node to estimate its own position based on limited exchange of information in the network. Our framework encompasses the case of sporadic measurements and random link failures. We prove the consistency of our algorithm in the case of fixed sensors. Finally, we provide numerical and experimental results from both simulated and real data. Simulations issued to real data are conducted on a wireless sensor network testbed.
\end{abstract}

Keywords: Principal component analysis, Wireless sensor networks, Distributed stochastic approximation algorithms, Localization, Multidimensional scaling, Received signal strength indicator

\footnotetext{
Email addresses: morraladetelecom-paristech.fr(G. Morral), bianchi@telecom-paristech.fr(P. Bianchi)

${ }^{1}$ The work of G. Morral is supported by DGA (French Armement Procurement Agency) and the Institut Mines-Télécom. This work has been supported by the ANR grant ODISSEE.
} 


\section{Introduction}

The problem of self-localization involving low-cost radio devices in WSN can be viewed as an example of the internet of things (IoT). The evolution in the last 50 years of the embedded systems and smart grids has contributed to enable the WSN integrates the emerging system of the IoT. Recently, advanced applications to handle specific tasks require the support of networking features to design cloudbased architectures involving sensor nodes, computers and other remote component. Among the large range of applications, location services can be provided by small devices carried by persons or deployed in a given area, e.g. routing and querying purposes, environmental monitoring, home automation services.

In this paper we investigate the problem of localization in wireless sensor networks (WSN) as a particular application of principal component analysis (PCA). We assume that wireless sensor devices are able to obtain received signal strength indicator (RSSI) measurements that can be related to a ranging model depending on the inter-sensor distances. The multidimensional scaling mapping method (MDSMAP) consists in applying PCA to a so-called similarity matrix constructed from the squared inter-sensor distances. Then, the sensors' positions can be recovered (up to a rigid transformation) from the principal components of the similarity matrix [1], [2]. As opposed to time difference of arrival (TDOA) and angle of arrival (AOA) techniques, the MDS-MAP approach allows to recover the network configuration based on the sole RSSI, and can be used without any additional hardware or/and synchronization specifically devote to self-localization.

MDS-MAP has been extensively studied in the literature (see Section2.3 for an 
overview). The algorithm is generally implemented in a centralized fashion. This requires the presence of a fusion center which gather sensors' measurements, computes the similarity matrix, performs the PCA, and eventually sends the positions to the respective sensors. In this paper, we provide a fully distributed algorithm which do not require RSSI measurements to be shared. In addition, our algorithm can be used on-line. By on-line, we mean that the current estimates of the sensors' positions are updated each time new RSSI measurements are performed, as opposed to batch methods which assume that measurements are collected prior to the localization step. Therefore, although we assume throughout the paper that the sensors' positions are fixed, our algorithm has the potential to be generalized to moving sensors, with aim to track positions while sensors are moving.

The paper is organized as follows. In Section 2, we provide the network and the observation models. We also provide a brief overview of standard self-localization techniques for WSN. Section 3 presents the centralized version of the MDS-MAP algorithm. The proposed distributed MDS-MAP algorithm is provided in Section 4 An additional refinement phase is also proposed in Section 5 where our MDS-MAP algorithm is coupled with a distributed maximum-likelihood estimator. In Section 6, numerical experiments based on both simulated and real data are provided. Section 7 gives some concluding remarks.

\section{The framework}

\subsection{Network model}

Consider $N$ agents (e.g. sensor nodes or other electronic devices) seeking to estimate their respective positions defined as $\left\{\boldsymbol{z}_{1}, \cdots, \boldsymbol{z}_{N}\right\}$ where for any $i, \boldsymbol{z}_{i} \in$ $\mathbb{R}^{p}$ with $p=2$ or 3 . We assume that agents have only access to noisy measurements 
of their relative RSSI values. More precisely, each agent $i$ observes some RSSI measurements $P_{i, j}$ associated with other agents $j \neq i$. Here, $P_{i, j}$ is a random function of the Euclidean distance $d_{i, j}=\left\|\boldsymbol{z}_{i}-\boldsymbol{z}_{j}\right\|$ between nodes $i$ and $j$. The statistical model relating RSSI values to inter-sensor distances is provided in the next paragraph.

The goal is to design a distributed and on-line algorithm to enable each sensor node to estimate its position $\boldsymbol{z}_{i}$ from noisy measurements of the distances. Before going further in the description of the RSSI statistical model, it is worth noting that the localization problem is in fact ill-posed. Since the only input data are distances, exact positions are identifiable only up to a rigid transformation. Indeed, quantities $\left(d_{i, j}\right)_{\forall i, j}$ are preserved when an isometry is applied to the agents' positions, i.e. rotation and translation. The problem is generally circumvented by assuming a minimum number of anchors or also named landmarks (sensor nodes whose GPS-positions are known), e.g. $M=3$ or 4 when $p=2$, and considering these prior knowledge to identify the indeterminacy. This point is further discussed in Section 2.3 .

\subsection{Received signal model}

We rely on the so-called log-normal shadowing model (LNSM) to model RSSI measurements as a function of the inter-sensor distance [3]. We define the average path loss $\mathrm{PL}(d)$ at a distance $d$ expressed in $\mathrm{dB}$ as $\mathrm{PL}(d)=\mathrm{PL}_{0}+10 \eta \log _{10} \frac{d}{d_{0}}$, where the parameters $\eta, d_{0}$ and $\mathrm{PL}_{0}$ depend on the environment (see Section 6). Given that the distance between sensors $i$ and $j$ is $d_{i, j}$, we define the RSSI between 
$i$ and $j$ as a random variable $P_{i, j}$ satisfying

$$
P_{i, j}=-\mathrm{PL}\left(d_{i, j}\right)+\epsilon_{i, j}
$$

where $\left(\epsilon_{i, j}: i \neq j\right)$ are thermal noises assumed independent with zero mean and variance $\sigma^{2}$. Assume that a given agent $i$ is provided with $T$ independent copies $P_{i, j}(1), \ldots, P_{i, j}(T)$ of the random variable $P_{i, j}$ and let $\bar{P}_{i, j}=T^{-1} \sum_{t=1}^{T} P_{i, j}(t)$ be the empirical average. An unbiased estimate of the squared distance $d_{i, j}^{2}$ is given by

$$
D(i, j)={\frac{10}{C^{4}}}^{\frac{-\bar{P}_{i, j}-\mathrm{PL}_{0}}{5 \eta}}
$$

where $C=10^{\frac{\sigma^{2} \ln 10}{2 T(10 \eta)^{2}}}$. Indeed, it can be easily checked that the mean and variance of the unbiased estimator (2) are respectively: $\mathbb{E}[D(i, j)]=d_{i, j}^{2}$ and $\mathbb{E}[(D(i, j)-$ $\left.\left.d_{i, j}^{2}\right)^{2}\right]=d_{i, j}^{4}\left(C^{8}-1\right)$. The construction of unbiased estimates of squared distance will be the basic ingredient of our distributed MDS-MAP algorithm.

\subsection{Overview of some localization techniques}

Several overview papers have been published in the last ten years dealing with the classification of the localization techniques (see [4] or [5]). In some situations, localization is made easier by the presence of anchor-nodes whose positions are assumed perfectly known. Other methods, called anchor-free, do not require the presence of such landmarks.

Anchor-based methods: The classical techniques involve the resolution of a single unknown position of a sensor node at a time by means of RSSI values following the LNSM coming from a fixed number of surrounding anchor nodes or landmarks. Since the sensor node only uses the information from known posi- 
tions, its position can be expressed in absolute coordinates, i.e. anchor positions in GPS-coordinates. When considering a noisy scenario, several works coupled the classical methods (trilateration, multilateration [6] ormin-max [7]) with a least squares problem. In particular, [8], [9] and [7] consider multi-hop communications between the sensor nodes. Other approaches focus on the statistical distribution of the received RSSI measurements coming from the landmarks. The goal is to consider a parametric model for the received signal and to apply maximum likelihood estimator (MLE). Most works consider the LNSM (see for instance [10] or [11]) while others assume alternative statistical models (see [12] or [13]).

Anchor-free methods: The configuration of the network can be recovered on a relative coordinate system instead of the GPS absolute coordinate system. When distances between nodes are view as similarity metrics, the positioning problem is referred to multidimensional scaling (MDS). The aim is to find an embedding from the $N$ nodes such that distances are preserved. In classical MDS [1, Chapter 12] positions are obtained by principal component analysis (PCA) of a $N \times N$ matrix constructed from the Euclidean distances. If distances are issued to some noise, e.g. estimated from RSSI measurements as (2), [2] propose a MDS-MAP algorithm based on the classical MDS problem. Indeed, the WSN localization problem is solved by enabling each sensor node to infer all the estimated pairwise distances. Alternative approaches within the localization context are based on optimization techniques. In metric MDS, positions are obtained by the stress majorization algorithm SMACOF (see [1, Chapter 8] and [14]). Alternatively, semidefinite programming (SDP) can be used as in [15].

The latter approaches have been also addressed in a distributed setting without the presence of a central processing unit. A distributed batch version of the 
SMACOF algorithm based on a round-robin communication scheme is proposed in [16]. Since [16] considers the minimization of the non-convex stress function, the same distributed approach (batch and incremental) is presented in [17] but using a quadratic criterion which includes the information from the anchor nodes to overcome the non-convex issue. The Authors of [15] propose a distributed implementation of their SDP-based localization algorithm. In [18] the network is divided in several clusters of at least two anchor nodes and a large number of sensor nodes and then the SDP problem is addressed locally at each cluster. More recently, gossip-based algorithms have been proposed in [19], [20] to solve the distributed optimization problem via Kalman filtering and gradient descent approaches. Other works address the distributed WSN localization problem using the multidimensional scaling (MDS) method based on PCA. The MDS-MAP proposed in [2] is later improved in [21]. In [21] each sensor node applies the MDS-MAP of [2] to its local map and then the local maps are merged sequentially to recover the global map. Alternatively, in [22] and [23] a sparsification matrix model on the observations is introduced to decentralized the PCA step.

\section{Centralized MDS-MAP}

\subsection{Centralized batch MDS}

Define $\boldsymbol{S}$ as the $N \times N$ matrix of square relative distances i.e., $\boldsymbol{S}(i, j)=d_{i, j}^{2}$.

Define $\overline{\boldsymbol{z}}=\frac{1}{N} \sum_{i=1}^{N} \boldsymbol{z}_{i}$ as the center of mass (or barycenter) of the agents. Upon noting that $d_{i, j}^{2}=\left\|\boldsymbol{z}_{i}-\overline{\boldsymbol{z}}\right\|^{2}+\left\|\boldsymbol{z}_{j}-\overline{\boldsymbol{z}}\right\|^{2}-2\left\langle\boldsymbol{z}_{i}-\overline{\boldsymbol{z}}, \boldsymbol{z}_{j}-\overline{\boldsymbol{z}}\right\rangle$, one has:

$$
\boldsymbol{S}=\boldsymbol{c} \mathbf{1}^{T}+\mathbf{1} \boldsymbol{c}^{T}-2 \boldsymbol{Z} \boldsymbol{Z}^{T}
$$


where 1 is the $N \times p$ matrix whose components are all equal to one, $\boldsymbol{c}=\left(\| z_{1}-\right.$ $\left.\overline{\boldsymbol{z}}\left\|^{2}, \cdots,\right\| \boldsymbol{z}_{N}-\overline{\boldsymbol{z}} \|^{2}\right)^{T}$ and the $i$ th line of matrix $\boldsymbol{Z}$ coincides with the row-vector $\boldsymbol{z}_{i}-\overline{\boldsymbol{z}}$. Otherwise stated, the $i$ th line of $\boldsymbol{Z}$ coincides with the barycentric coordinates of node $i$. Define $\boldsymbol{J}=\mathbf{1 1}^{T} / N$ as the orthogonal projector onto the linear span of the vector $\mathbf{1}=(1, \ldots, 1)^{T}$. Define $\boldsymbol{J}_{\perp}=\boldsymbol{I}_{N}-\boldsymbol{J}$ as the projector onto the space of vectors with zero sum, where $\boldsymbol{I}_{N}$ is the $N \times N$ identity matrix. It is straightforward to verify that $J_{\perp} Z=Z$. Thus, introducing the matrix

$$
\boldsymbol{M} \triangleq-\frac{1}{2} J_{\perp} S J_{\perp}
$$

equation (3) implies that $\boldsymbol{M}=\boldsymbol{Z} \boldsymbol{Z}^{T}$. In particular, $\boldsymbol{M}$ is symmetric, non-negative and has rank (at most) $p$. The agents' coordinates can be recovered from $\boldsymbol{M}$ (up to a rigid transformation) by recovering the principal eigenspace of $M$ i.e., the vector-space spanned by the $p$ th principal eigenvectors (see [1, Chapter 12]).

Denote by $\left\{\lambda_{k}\right\}_{k=1}^{N}$ the eigenvalues of $\boldsymbol{M}$ in decreasing order, i.e. $\lambda_{1} \geq \cdots \geq$ $\lambda_{N}$. In the sequel, we shall always assume that $\lambda_{p}>0$. Denote by $\left\{\boldsymbol{u}_{k}\right\}_{k=1}^{p}$ corresponding unit-norm $N \times 1$ eigenvectors. Set $\boldsymbol{Z}=\left(\sqrt{\lambda_{1}} \boldsymbol{u}_{1}, \cdots, \sqrt{\lambda_{p}} \boldsymbol{u}_{p}\right)$. Clearly $\boldsymbol{M}=\boldsymbol{Z} \boldsymbol{Z}^{T}=\bar{Z} \bar{Z}$ and $\bar{Z}=\boldsymbol{R} \boldsymbol{Z}$ for some matrix $\boldsymbol{R}$ such that $\boldsymbol{R} \boldsymbol{R}^{T}=$ $\boldsymbol{I}_{N}$. Otherwise stated, $\bar{Z}$ coincides with the barycentric coordinates $\boldsymbol{Z}$ up to an orthogonal transformation. In particular, the $i$ th row of matrix $\bar{Z}$ is an estimate of the position of the $i$ th sensor (up to the latter transformation common to all sensors). In practice, matrix $S$ is usually not perfectly known and must be replaced by an estimate $\widehat{S}$. This yields the Algorithm 1 (see [1, Chapter 12]). 
Algorithm 1: Centralized batch MDS-MAP for localization

Input: Noisy estimates of the square distances $D(i, j)$ (2) for all pair $i, j$.

1. Compute matrix $\widehat{\boldsymbol{S}}=(D(i, j))_{i, j=1, \ldots, N}$.

2. Set $\widehat{\boldsymbol{M}}=-\frac{1}{2} \boldsymbol{J}_{\perp} \widehat{\boldsymbol{S}} \boldsymbol{J}_{\perp}$.

3. Find the eigenvectors $\left\{\boldsymbol{u}_{k}\right\}_{k=1}^{p}$ and eigenvalues $\left\{\lambda_{k}\right\}_{k=1}^{p}$ of $\widehat{\boldsymbol{M}}$.

Output: $\widehat{\boldsymbol{Z}}=\left(\sqrt{\lambda_{1}} \boldsymbol{u}_{1}, \cdots, \sqrt{\lambda_{p}} \boldsymbol{u}_{p}\right)$

\subsection{Centralized on-line $M D S$}

In the previous batch Algorithm 1 measurements are made prior to the estimation of the coordinates. From now on, observations are not stored into the system's memory: they are deleted after use. Thus, agents gather measurements of their relative distance with other agents and, simultaneously, estimate their position.

\subsubsection{Observation model: sparse measurements}

We introduce a collection of independent r.v.'s $\left(P_{i, j}(n): i, j=1, \cdots, N, n \in\right.$ $\mathbb{N})$ such that each $P_{i, j}(n)$ follows the LNSM described in Section 2.2 At time $n$, it is possible to define an unbiased estimate $\boldsymbol{D}_{n}(i, j)$ the squared distance as $\boldsymbol{D}_{n}(i, j)=\frac{10}{C^{4}} \frac{-P_{i, j}(n)-\mathrm{PL}_{0}}{5 \eta}$ in the sense that $\mathbb{E}\left[\boldsymbol{D}_{n}(i, j)\right]=d_{i, j}^{2}$. We use the convention that $\boldsymbol{D}_{n}(i, i)=0$.

Definition 1 (Sparse measurements). At each time instant $n$, we assume that with probability $q_{i j}$, an agent $i$ is able to obtain an estimate $\boldsymbol{S}_{n}(i, j)$ of the square distance with an other agent $j \neq i$ and makes no observation otherwise. Thus, one can represent the available observations as the product $\boldsymbol{S}_{n}(i, j)=\boldsymbol{A}_{n}(i, j) \boldsymbol{D}_{n}(i, j)$ where $\left(\boldsymbol{A}_{n}\right)_{n}$ is an i.i.d. sequence of random matrices whose components $\boldsymbol{A}_{n}(i, j)$ follow the Bernoulli distribution of parameter $q_{i j}$. Stated otherwise, node $i$ observes the ith row of matrix $\boldsymbol{A}_{n} \circ \boldsymbol{D}_{n}$ at time $n$ where $\circ$ stands for the Hadamard product. 
Lemma 1. Assume $q_{i j}>0$ for all pairs $i, j$. Set $\boldsymbol{W}:=\left[q_{i j}^{-1}\right]_{i, j=1}^{N}$ and let $\boldsymbol{A}_{n}, \boldsymbol{S}_{n}$ be defined as above. The matrix

$$
\boldsymbol{S}_{n}=\boldsymbol{W} \circ \boldsymbol{A}_{n} \circ \boldsymbol{D}_{n}
$$

is an unbiased estimate of $\boldsymbol{S}$ i.e., $\mathbb{E}\left[\boldsymbol{S}_{n}\right]=\boldsymbol{S}$.

Proof. Each entry of matrix $\boldsymbol{S}_{n}, \boldsymbol{S}_{n}(i, j)$, is equal to $1 / q_{i j} \boldsymbol{A}_{n}(i, j) \boldsymbol{D}_{n}(i, j)$. As the random variables $\boldsymbol{A}_{n}(i, j)$ and $\boldsymbol{D}_{n}(i, j)$ are independent, by the above definition of $\boldsymbol{D}_{n}$ and $\mathbb{E}\left[\boldsymbol{A}_{n}(i, j)\right]=q_{i j}$, then $\mathbb{E}\left[\boldsymbol{S}_{n}(i, j)\right]=d_{i, j}^{2}$.

As a consequence of Lemma 1, an unbiased estimate of $M$ defined in (4) is simply obtained by $\boldsymbol{M}_{n}=-\frac{1}{2} \boldsymbol{J}_{\perp} \boldsymbol{S}_{n} \boldsymbol{J}_{\perp}$.

\subsubsection{Oja's algorithm for the localization problem}

When dealing with random matrices $\boldsymbol{M}_{n}$ having a given expectation $\boldsymbol{M}$, the principal eigenspace of $M$ can be recovered by the Oja's algorithm [24]. The latter consists in recursively defining a sequence $\boldsymbol{U}_{n}$ of $N \times p$ matrices, which stand for the estimate at time $n$ of the $p$ principal unit-eigenvectors of $M$. The iterations as firstly introduced in [24] are given by:

$$
\boldsymbol{U}_{n}=\boldsymbol{U}_{n-1}+\gamma_{n}\left(\boldsymbol{M}_{n} \boldsymbol{U}_{n-1}-\boldsymbol{U}_{n}\left(\boldsymbol{U}_{n-1}^{T} \boldsymbol{M}_{n} \boldsymbol{U}_{n-1}\right)\right)
$$

where $\gamma_{n}>0$ is a step size. Note that in practice, the algorithm is likely to suffer from numerical instabilities. In [25], a renormalization step is introduced to avoid unstabilities. As this approach seems difficult to generalize in a distributed context, 
it is more adequate in our context to introduce a reprojection step in (6) of the form

$$
\boldsymbol{U}_{n}=\Pi_{\mathcal{K}}\left[\boldsymbol{U}_{n-1}+\gamma_{n}\left(\boldsymbol{M}_{n} \boldsymbol{U}_{n-1}-\boldsymbol{U}_{n}\left(\boldsymbol{U}_{n-1}^{T} \boldsymbol{M}_{n} \boldsymbol{U}_{n-1}\right)\right)\right]
$$

where $\Pi_{\mathcal{K}}$ is a projector onto an arbitrarily large convex compact set $\mathcal{K}$ chosen large enough to include all matrices whose columns have unit-norm. Typically, we set $\mathcal{K}=[-\alpha, \alpha]^{p} \times \cdots \times[-\alpha, \alpha]^{p}$ where $\alpha>1$.

In order to obtain an estimate of the sensors positions, we also need to estimate the principal eigenvalues in addition to the eigenvectors. Let $\boldsymbol{u}_{n, k}$ denote the $k$ th column of matrix $\boldsymbol{U}_{n}$. Define the quantity $\lambda_{n, k}$ recursively by:

$$
\lambda_{n, k}=\lambda_{n-1, k}+\gamma_{n}\left(\boldsymbol{u}_{n-1, k}^{T} \boldsymbol{M}_{n} \boldsymbol{u}_{n-1, k}-\lambda_{n-1, k}\right) .
$$

The convergence properties of Oja's algorithm are studied in details in [24] and [25]. Finally, according to step 3 of the batch Algorithm 1 the estimated barycentric coordinates are obtained as:

$$
\widehat{Z}_{n}=\left(\sqrt{\lambda_{n, 1}} \boldsymbol{u}_{n, 1}, \ldots, \sqrt{\lambda_{n, p}} \boldsymbol{u}_{n, p}\right)
$$

The combination of Equations (6) (7) and (8) provides an on-line for MDS-MAP algorithm. However, the computation of matrix $\boldsymbol{M}_{n}$ at each step as well as the matrix products in (6) require a full amount of centralization. 


\section{Distributed on-line MDS-MAP}

\subsection{Communication model}

It is clear from the previous section that an unbiased estimate of matrix $M$ is the first step needed to estimate the sought eigenspace. In the centralized setting, this estimate was given by matrix $\boldsymbol{M}_{n}=-\frac{1}{2} \boldsymbol{J}_{\perp} \boldsymbol{S}_{n} \boldsymbol{J}_{\perp}$. As made clear by the observation model (in Definition 1), each node $i$ observes the $i$ th row of matrix $\boldsymbol{S}_{n}$. As a consequence, node $i$ has access to the $i$ th row-average $\overline{\boldsymbol{S}}_{n}(i) \triangleq$ $\frac{1}{N} \sum_{j} \boldsymbol{S}_{n}(i, j)$. This means that matrix $\boldsymbol{S}_{n} \boldsymbol{J}_{\perp}$ can be obtained with no need to further exchange of information in the network. On the other hand, $\boldsymbol{J}_{\perp} \boldsymbol{S}_{n} \boldsymbol{J}_{\perp}$ requires to compute the per-column averages of matrix $\boldsymbol{S}_{n} \boldsymbol{J}_{\perp}$, i.e. $\frac{1}{N} \sum_{j} \boldsymbol{S}_{n}(j, i)$ for all $i$. This task is difficult in a distributed setting, as it would require that all nodes share all their observations at any time. A similar obstacle happens in Oja's algorithm when computing matrix products, e.g. $\boldsymbol{M}_{n} \boldsymbol{U}_{n-1}$ in (6). To circumvent the above difficulties, we introduce the following sparse asynchronous communication framework. In order to derive an unbiased estimate of $M$, let us first remark that for all $i, j$,

$$
\boldsymbol{M}(i, j)=\frac{\overline{d^{2}}(i)+\overline{d^{2}}(j)}{2}-\frac{d_{i, j}^{2}+\delta}{2}
$$

where we set $\overline{d^{2}}(i) \triangleq \frac{1}{N} \sum_{k} d_{i k}^{2}$ and $\delta \triangleq \frac{1}{N} \sum_{i} \overline{d^{2}}(i)$. Note that the terms $d_{i, j}^{2}$ and $\overline{d^{2}}(i)$ can be estimated by $\boldsymbol{S}_{n}(i, j)$ and $\overline{\boldsymbol{S}}_{n}(i)$ respectively. However, additional communication is needed to estimate $\delta$ since it corresponds to the average value over all square distances. We define

$$
\widehat{\boldsymbol{M}}_{n}(i, j)=\frac{\overline{\boldsymbol{S}}_{n}(i)+\overline{\boldsymbol{S}}_{n}(j)}{2}-\frac{\boldsymbol{S}_{n}(i, j)+\boldsymbol{\delta}_{n}(i)}{2}
$$


where $\delta_{n}(i)$ is a quantity that we will define in the sequel, and which represents the estimate of $\delta$ at the agent $n$.

We are now faced with two problems. First, we must construct $\boldsymbol{\delta}_{n}(i)$ as an unbiased estimate of $\delta$. Second, we need to avoid the computation of $\widehat{\boldsymbol{M}}_{n}(i, j)$ for all pairs $i, j$, but only to some of them. In order to provide an answer to these problems, we introduce the notion of asynchronous transmission sequence. Formally,

Definition 2 (Asynchronous Transmission Sequence). Let $q$ be a real number such that $0<q<1$. We say that the sequence of random vectors $T_{n}=\left(\iota_{n}, Q_{n, i}\right.$ : $i \in\{1, \cdots, N\}, n \in \mathbb{N}$ ) is an Asynchronous Transmission Sequence (ATS) if: i) all variables $\left(\iota_{n}, Q_{n, i}\right)_{i, n}$ are independent, ii) $\iota_{n}$ is uniformly distributed on the set $\{1, \cdots, N\}$, iii) $\forall i \neq \iota_{n}, Q_{n, i}$ is a Bernoulli variable with parameter $q$ i.e., $\mathbb{P}\left[Q_{n, i}=1\right]=q$ and iv) $Q_{n, \iota_{n}}=0$.

Let $\left(T_{n}\right)_{n}$ denote an ATS defined as above. At time $n$, we assume that a given node $\iota_{n} \in\{1, \ldots, N\}$ wakes up and transmits its local row-average $\overline{\boldsymbol{S}}_{n}\left(\iota_{n}\right)$ to other nodes. All nodes $i$ such that $Q_{n, i}=1$ are supposed to receive the message. For any $i$, we set:

$$
\boldsymbol{\delta}_{n}(i)=\frac{\overline{\boldsymbol{S}}_{n}(i)}{N}+\frac{\overline{\boldsymbol{S}}_{n}\left(\iota_{n}\right) Q_{n, i}}{q}
$$

The following Lemma is a consequence of Definition 2 along with Lemma 1 and equation (4).

Lemma 2. Assume that $\left(T_{n}\right)_{n}$ is an ATS independent of $\left(\boldsymbol{S}_{n}\right)_{n}$. Let $\left(\widehat{\boldsymbol{M}}_{n}\right)_{n}$ be the sequence of matrices defined by (10). Then, $\mathbb{E}\left[\widehat{\boldsymbol{M}}_{n}\right]=M$.

Proof. By Lemma 1 the expectation of terms $\overline{\boldsymbol{S}}_{n}(i), \overline{\boldsymbol{S}}_{n}(j)$ and $\boldsymbol{S}_{n}(i, j)$ are respectively $\overline{d^{2}}(i), \overline{d^{2}}(j)$ and $d_{i, j}^{2}$. Moreover, by Definition 2 the expectation of the 
random term $\boldsymbol{\delta}_{n}(i)$ is equal to

$$
\mathbb{E}\left[\boldsymbol{\delta}_{n}(i)\right]=\frac{1}{N} \mathbb{E}\left[\overline{\boldsymbol{S}}_{n}(i)\right]+\frac{1}{q} \frac{1}{N} \sum_{j \neq i} \mathbb{E}\left[\overline{\boldsymbol{S}}_{n}(j)\right] q=\frac{1}{N} \sum_{i=1}^{N} \overline{d^{2}}(i)
$$

which coincides with $\delta$. Then, the expectation of each entry of the matrix $\widehat{\boldsymbol{M}}_{n}$ in (10) is equal to the corresponding $\boldsymbol{M}(i, j)$ defined in (9).

\subsection{Preliminaries: constructing unbiased estimates}

As we now obtain a distributed and unbiased estimate of $\boldsymbol{M}$, the remaining task is to adapt accordingly the Oja's algorithm (6). In this paragraph, we provide the main ideas behind the construction of our algorithm.

Assume that we are given a current estimate $\boldsymbol{U}_{n-1}$ at time $n$, under the form of a $N \times p$ matrix. Assume also that for each $i$, the $i$ th row of $\boldsymbol{U}_{n-1}$ is a variable which is physically handled by node $i$. We denote by $\boldsymbol{U}_{n-1}(i)$ the $i$ th row of $\boldsymbol{U}_{n-1}$.

Looking at (6) in more details, Oja's algorithm requires the evaluation of intermediate values, as unbiased estimates of $\boldsymbol{M} \boldsymbol{U}_{n-1}$ and $\boldsymbol{U}_{n-1}^{T} \boldsymbol{M} \boldsymbol{U}_{n-1}$.

We consider the previous ATS $\left(T_{n}\right)_{n}$ involved in (10). We assume that the active node $\iota_{n}$ (i.e., the one which transmits $\overline{\boldsymbol{S}}_{n}\left(\iota_{n}\right)$ ) is also able to transmit its local estimate $\boldsymbol{U}_{n-1}\left(\iota_{n}\right)$ at same time. Thus, with probability $\frac{1}{N}$, node $\iota_{n}$ sends its former estimate $\boldsymbol{U}_{n-1}\left(\iota_{n}\right)$ and $\overline{\boldsymbol{S}}_{n}\left(\iota_{n}\right)$ to all nodes $i$ such that $Q_{n, i}=1$. Then, all nodes compute:

$$
\boldsymbol{Y}_{n}(i)=\widehat{\boldsymbol{M}}_{n}(i, i) \boldsymbol{U}_{n-1}(i)+\frac{N}{q} \boldsymbol{U}_{n-1}\left(\iota_{n}\right) \widehat{\boldsymbol{M}}_{n}\left(i, \iota_{n}\right) Q_{n, i}
$$

As it will be made clear below, the $N \times p$ matrix $\boldsymbol{Y}_{n}$ whose $i$ th row coincides with $\boldsymbol{Y}_{n}(i)$ can be interpreted as an unbiased estimate of $\boldsymbol{M} \boldsymbol{U}_{n-1}$. 
Now we introduce the distributed version of the second term $\boldsymbol{U}_{n-1}^{T} \boldsymbol{M}_{n} \boldsymbol{U}_{n-1}$. Consider a second ATS $\left(T_{n}^{\prime}\right)_{n}$ independent of $\left(T_{n}\right)_{n}$. At time $n$, node $\iota_{n}^{\prime}$ wakes up uniformly random and broadcasts the product $\boldsymbol{U}_{n-1}\left(\iota_{n}^{\prime}\right)^{T} \boldsymbol{Y}_{n}\left(\iota_{n}^{\prime}\right)$ to other nodes. Receiving nodes are those $i$ 's for which $Q_{n, i}^{\prime}=1$. Then, all nodes are able to compute the estimate $p \times p$ matrix as follows:

$$
\boldsymbol{\Lambda}_{n}(i)=\boldsymbol{U}_{n-1}(i)^{T} \boldsymbol{Y}_{n}(i)+\frac{N}{q} \boldsymbol{U}_{n-1}\left(\iota_{n}^{\prime}\right)^{T} \boldsymbol{Y}_{n}\left(\iota_{n}^{\prime}\right) Q_{n, i}^{\prime} .
$$

Lemma 3. Let $\left(T_{n}\right)_{n}$ and $\left(T_{n}^{\prime}\right)_{n}$ be two independent ATS. For any $n$, denote by $\mathcal{F}_{n}$ the $\sigma$-field generated by $\left(T_{k}\right)_{k \leq n},\left(T_{k}^{\prime}\right)_{k \leq n},\left(A_{k}\right)_{k \leq n}$ and $\left(D_{k}\right)_{k \leq n}$. Let $\left(\boldsymbol{U}_{n}\right)_{n}$ be a $\mathcal{F}_{n}$-measurable $N \times p$ random matrix and let $\boldsymbol{Y}_{n}, \boldsymbol{\Lambda}_{n}$ be defined as above. Then,

$$
\mathbb{E}\left[\boldsymbol{Y}_{n} \mid \mathcal{F}_{n-1}\right]=\boldsymbol{M} \boldsymbol{U}_{n-1} \quad \text { and } \quad \mathbb{E}\left[\boldsymbol{\Lambda}_{n}(i) \mid \mathcal{F}_{n-1}\right]=\boldsymbol{U}_{n-1}^{T} \boldsymbol{M} \boldsymbol{U}_{n-1}
$$

Under Lemma 12 and Definition 2 the random sequences $\boldsymbol{Y}_{n}(i)$ and $\boldsymbol{\Lambda}_{n}(i)$ are unbiased estimates of $\sum_{j} \boldsymbol{M}(i, j) \boldsymbol{U}_{n-1}(j)$ and $\boldsymbol{U}_{n-1}^{T} \boldsymbol{M} \boldsymbol{U}_{n-1}$ respectively given $\boldsymbol{U}_{n-1}$.

Proof. For each $i$, we obtain

$$
\begin{aligned}
\mathbb{E}\left[\boldsymbol{Y}_{n}(i) \mid \mathcal{F}_{n-1}\right] & =\boldsymbol{M}(i, i) \boldsymbol{U}_{n-1}(i)+\frac{N}{q} \frac{q}{N} \sum_{j \neq i} \boldsymbol{M}(i, j) \boldsymbol{U}_{n-1}(j) \\
& =\sum_{j} \boldsymbol{M}(i, j) \boldsymbol{U}_{n-1}(j)
\end{aligned}
$$


and

$$
\begin{aligned}
\mathbb{E}\left[\boldsymbol{\Lambda}_{n}(i) \mid \mathcal{F}_{n-1}\right] & =\boldsymbol{U}_{n-1}(i)^{T} \mathbb{E}\left[\boldsymbol{Y}_{n}(i) \mid \mathcal{F}_{n-1}\right]+\frac{N}{q} \frac{1}{N} \sum_{j \neq i} \boldsymbol{U}_{n-1}(j)^{T} \mathbb{E}\left[\boldsymbol{Y}_{n}(j) \mid \mathcal{F}_{n-1}\right] q \\
& =\sum_{i} \sum_{j} \boldsymbol{U}_{n-1}(i)^{T} \boldsymbol{M}(i, j) \boldsymbol{U}_{n-1}(j)
\end{aligned}
$$

which corresponds with the square matrix $\boldsymbol{U}_{n-1}^{T} \boldsymbol{M} \boldsymbol{U}_{n-1}$.

\subsubsection{Main algorithm}

We are now ready to state the main algorithm. The algorithm generates iteratively and for any node $i$ two variables $\boldsymbol{U}_{n}(i)$ and $\boldsymbol{\lambda}_{n}(i)$, according to:

$$
\begin{aligned}
& \boldsymbol{U}_{n}(i)=\boldsymbol{U}_{n-1}(i)+\gamma_{n}\left(\boldsymbol{Y}_{n}(i)-\boldsymbol{U}_{n-1}(i) \boldsymbol{\Lambda}_{n}(i)\right) \\
& \boldsymbol{\lambda}_{n}(i)=\boldsymbol{\lambda}_{n-1}(i)+\gamma_{n}\left(\operatorname{diag}\left(\boldsymbol{\Lambda}_{n}(i)\right)-\boldsymbol{\lambda}_{n-1}(i)\right)
\end{aligned}
$$

For the same reasons as before, it is important in practice to introduce a projection step $\Pi_{\mathcal{K}}$ in (14) to avoid numerical unstabilities. Finally, as in (8), each sensor $i$ obtains its estimate position $\widehat{Z}_{n}(i)$ by:

$$
\widehat{\boldsymbol{Z}}_{n}(i)=\left(\sqrt{\boldsymbol{\lambda}_{n, 1}(i)} \boldsymbol{u}_{n, 1}(i), \cdots, \sqrt{\boldsymbol{\lambda}_{n, p}(i)} \boldsymbol{u}_{n, p}(i)\right)
$$

where we set $\boldsymbol{U}_{n}(i)=\left(u_{n, 1}(i), \ldots, \boldsymbol{u}_{n, p}(i)\right)$.

The proposed algorithm (14)-(16) is summarized in Algorithm 2 below. Note that, at each iteration time $n$, only two communications are performed by two randomly selected nodes issued to the ATS's $T_{n}$ and $T_{n}^{\prime}$. 
Algorithm 2: Distributed on-line MDS-MAP for localization (doMDS)

Update: At each time $n=1,2, \ldots$

[Measures]: each sensor node $i$, do:

Makes sparse measurements of their RSSI to obtain $\left(\boldsymbol{D}_{n}(i, j)\right)_{j}$ for some $j$ such that $\boldsymbol{A}_{n}(i, j)=1$ (Definition 1). Set

$$
\boldsymbol{S}_{n}(i, j)= \begin{cases}q_{i j}^{-1} \boldsymbol{D}_{n}(i, j) & \text { if } \boldsymbol{A}_{n}(i, j)=1 \\ 0 & \text { otherwise }\end{cases}
$$

and set $\overline{\boldsymbol{S}}_{n}(i)=\frac{1}{N} \sum_{j} \boldsymbol{S}_{n}(i, j)$.

[Communication step]:

A randomly selected node $\iota_{n}$ wakes up, then

i) The node $\iota_{n}$ randomly selected broadcasts $\boldsymbol{U}_{n-1}\left(\iota_{n}\right)$ and $\overline{\boldsymbol{S}}_{n}\left(\iota_{n}\right)$ to nodes $i$ such that $Q_{n, i}=1$.

ii) Each node $i$ computes $\boldsymbol{Y}_{n}(i)$ by (12).

iii) A node $\iota_{n}^{\prime}$ randomly selected broadcasts $\boldsymbol{U}_{n-1}\left(\iota_{n}^{\prime}\right)^{T} \boldsymbol{Y}_{n}\left(\iota_{n}^{\prime}\right)$ to nodes $i$ such that $Q_{n, i}^{\prime}=1$.

iv) Each node $i$ updates $\boldsymbol{U}_{n}(i)$ by (13)-(14) and $\widehat{\boldsymbol{Z}}_{n}(i)$ by (16).

\subsection{Convergence analysis}

We make the following assumptions. The sequence $\left(\gamma_{n}\right)_{n}$ is positive and satisfies

$$
\sum_{n} \gamma_{n}=+\infty \quad \text { and } \quad \sum_{n} \gamma_{n}^{2}<\infty
$$

Moreover we make the assumption that the sequence $\boldsymbol{U}_{n}$ remains a.s. in a fixed compact set $\mathcal{K}$. It must be emphasized that this assumption is difficult to check in practice. As mentioned above, stability can be enforced by means of a projection step onto $\mathcal{K}$.

Proposition 1. For any $\boldsymbol{U} \in \mathbb{R}^{N \times p}$, set $h(\boldsymbol{U})=\boldsymbol{M U}-\boldsymbol{U} \boldsymbol{U}^{T} \boldsymbol{M U}$. Let $\boldsymbol{U}_{n}$ be defined by (14). There exists a random sequence $\xi_{n}$ such that, almost surely (a.s.), 
$\sum_{n} \gamma_{n} \xi_{n}$ converges and

$$
\boldsymbol{U}_{n}=\boldsymbol{U}_{n-1}+\gamma_{n} h\left(\boldsymbol{U}_{n-1}\right)+\gamma_{n} \xi_{n}
$$

The proof is provided in the Appendix. We are now in position to state the main convergence result.

Theorem 4. For any $k=1, \cdots, p$, the kth column $\boldsymbol{u}_{n, k}$ of $\boldsymbol{U}_{n}$ converges to an eigenvector of $\boldsymbol{M}$ with unit-norm. Moreover, for each node $i, \boldsymbol{\lambda}_{n, k}(i)$ converges to the corresponding eigenvalue.

The proof is provided in the Appendix.

Note that Theorem 4 might seem incomplete in some respect: one indeed expects that the sequence $U_{n}$ converges to the principal eigenspace of $\boldsymbol{M}$. Instead, Theorem 4 only guarantees that one recovers some eigenspace of $\boldsymbol{M}$. Undesired limit points can be theoretically avoided by introducing an arbitrary small Gaussian noise inside the parenthesis of the left hand side of (14) (see Chapter 4 in [26]). These so-called avoidance of traps techniques are however out of the scope of this paper, and numerical results indicate that the principal eigenspace is indeed recovered in practical situations.

\section{Position refinement: distributed maximum likelihood estimator}

In the context of WSN localization, a refinement phase is in general added (see [9], [7], [21] or [16]). It is usually based on the statistical model relating the observed RSSI values to the unknown positions, the latter being estimated in the maximum likelihood sense. The objective is twofolds. First, maximum likelihood 
estimation improves the estimation accuracy obtained by the MDS-MAP approach. Second, as the MDS-MAP only identifies positions up to a rigid transformation, it allows to eliminate the residual ambiguity by using anchor nodes, provided that such anchors exist.

In this section, we provide a distributed algorithm in order to locally maximize the likelihood. It is worth noting that the likelihood function is generally non-convex. Thus, one cannot expect that a standard gradient ascent provides the maximum likelihood estimator regardless from the initialization. For this reason, a preliminary phase such as the proposed doMDS algorithm is essential as an initial coarse estimate, and the algorithm depicted below is used merely as a fine search in the neighborhood of the doMDS output.

\subsection{Likelihood function}

Consider a connected graph $G=(V, E)$ where $V=\{1, \ldots, N\}$ is the set of agents and $E$ is a set of non-directed edges. In this paragraph, we allow for the presence of anchor nodes. We let $A \subset\{1, \ldots, N\}$ be the set of anchor nodes i.e. for each $k \in A$, the position $z_{k}$ of node $k$ is assumed to be known. Unknown parameters thus reduce to set of coordinates $\boldsymbol{z}=\left(\boldsymbol{z}_{i}: i \in \bar{A}\right)$ where $\bar{A}=V \backslash A$. We denote by $\mathscr{N}_{i}$ the neighbors of $i$ which belong to $\bar{A}$ and by $\mathscr{M}_{i}$ the neighbors of $i$ which are anchors. We note $\boldsymbol{z}_{\mathscr{N}_{i}}=\left(\boldsymbol{z}_{j}: j \in \mathscr{N}_{i} \cup\{i\}\right)$. For a connected pair of nodes $\{i, j\}$, we let $P_{i, j}(n)(n \in \mathbb{N})$ be an i.i.d. sequence following the LNSM model of Section 2.2 Equivalently, the quantity $\hat{\ell}_{i, j}(n)=\frac{-P_{i, j}(n)-\mathrm{PL}_{0}}{10 \eta}$ follows a normal distribution with mean $\log _{10} d_{i, j}$ and variance $\frac{\sigma^{2}}{100 \eta^{2}}$, since $\hat{\ell}_{i, j}(n)=\log _{10} d_{i, j}+\frac{\varepsilon_{i, j}}{10 \eta}$ by using (11). Based on the observations $\left(\ell_{i, j}(n): i \sim j\right)$ at a given time $n$, the 
likelihood associated with the unknown sensors' positions can be decomposed as

$$
\mathcal{L}_{n}(\boldsymbol{z})=\sum_{i=1}^{N} f_{i, n}\left(\boldsymbol{z}_{\mathscr{N}_{i}}\right)
$$

where

$f_{i}\left(\boldsymbol{z}_{\mathscr{N}_{i}}\right)=\sum_{j \in \mathscr{N}_{i}}\left(\hat{\ell}_{i, j}(n)-\log _{10}\left\|\boldsymbol{z}_{i}-\boldsymbol{z}_{j}\right\|\right)^{2}+\sum_{k \in \mathscr{M}_{i}}\left(\hat{\ell}_{i k}(n)-\log _{10}\left\|\boldsymbol{z}_{i}-\boldsymbol{z}_{k}\right\|\right)^{2}$.

\subsection{The algorithm: on-line gossip-based implementation}

Following the idea of [27](see also [28] and reference therein), we propose a distributed consensus-based implementation consisting on local computations and random communications among the sensor nodes. The algorithm is given below. The convergence proof is omitted due to the lack of space but follows from the same arguments as [28].

\section{Algorithm 3: Distributed on-line MLE (doMLE)}

Update: at each time $n=1,2, \ldots$

[Local step] each node $i$ obtains $\left\{P_{i, j}(n)\right\}_{\forall j \in \mathscr{N}_{i}}$ and $\left\{P_{i k}(n)\right\}_{\forall k \in \mathscr{M}_{i}}$.

Each sensor $i$ computes a temporary estimate of its position's set:

$$
\tilde{\boldsymbol{z}}_{\mathscr{N}_{i}, n}=\boldsymbol{z}_{\mathscr{N}_{i}, n-1}-\gamma_{n} \nabla f_{i, n}\left(\boldsymbol{z}_{\mathscr{N}_{i}, n-1}\right)
$$

[Gossip step] two uniformly random selected nodes $i \sim j$ in $\bar{A}$ exchange their temporary estimated positions and average their values according to:

$$
\begin{aligned}
\forall \ell \in \mathscr{N}_{i} \cap \mathscr{N}_{j}, & \boldsymbol{z}_{\mathscr{N}_{i}, n}(\ell) \\
\boldsymbol{z}_{\mathscr{N}_{j}, n}(\ell) & =\frac{\tilde{\boldsymbol{z}}_{\mathscr{N}_{i}, n}(\ell)+\tilde{\boldsymbol{z}}_{\mathscr{N}_{j}, n}(\ell),}{2}
\end{aligned}
$$

Otherwise, $\forall \ell \notin \mathscr{N}_{i} \cap \mathscr{N}_{j}$ or $m \neq i, j$, set $\boldsymbol{z}_{\mathscr{N}_{m}, n}(\ell)=\tilde{\boldsymbol{z}}_{\mathscr{N}_{m}, n}(\ell)$.

Algorithm 3 uses a standard pairwise averaging between nodes. We note that more involved gossip protocols have been proposed, we mention for instance broad- 
cast and push-sum protocols (see [29] and [30]). Although theoretically possible, such an extension of Algorithm 3 is however beyond the scope of this paper.

\section{Numerical results}

We consider the same network configuration corresponding on the set of $N=$ 50 sensor nodes selected from the FIT IoT-LAB 2 platform at Rennes. Sensor nodes are located within a $5 \times 9 \mathrm{~m}^{2}$ area, i.e. $p=2$. Six sensors of the 50 were set as anchor nodes (or landmarks). We compare the performance of our proposed distributed on-line MDS (doMDS) to other existing algorithms. We consider the distributed batch MDS [16] (dwMDS) and the classical centralized methods such as: multilateration [6] (MC), min-max [7], Algorithm 1 in Section 3.1 (batch MDS) and the Oja's algoritm (6)-(7) described in Section 3.2 The three iterative algorithms (Oja's, dwMDS and doMDS) are initialized by randomly chosen positions in $5 \times 9 \mathrm{~m}^{2}$.

\subsection{Simulated data}

First, we show the results from simulated data drawn according to the observation model defined in Section 3.2. In order to compare our proposed algorithm with the distributed MDS proposed by [16], we set the same environmental context in which $\sigma / \eta=1.7$. Figure 1 displays the comparison of the root-mean square error (RMSE) when running Algorithm 2 over 300 independent runs of the estimated positions when considering different communication parameters: $\left(q_{i j}\right)_{i, j}$ (the Bernoullis related to the observation model (5)) and $q$ (the Bernoullis related

\footnotetext{
${ }^{2}$ FIT IoT-LAB https://www. iot-lab.info/
} 
to the ATS in Definition 22). Since the variance of the error sequence is upper bounded by the minimum probability value in A.3 - A.5, we observe from Figure 1 a trade-off between the accuracy and the number of communications as the RMSE decreases faster when the probability $q$ is closer to 1 .

Figure 2a shows the comparison of the localization RMSE over 300 independent runs of the overall estimated positions when considering the three iterative methods: the centralized Oja's [6)-(7), the dwMDS of [16] and our proposed Algorithm 2 The estimated positions after 1000 iterations of the three iterative algorithms are reported in Figure 2. Note that, the result in Figure $2 \mathrm{c}$ requires at least twice the number of communications compared to the results both on-line Oja's approaches. Positions close to the barycentric of the network tend to be more accurate than positions on the surrounding area for the three cases. Nevertheless, Figures $2 \mathrm{~b}$ and $2 \mathrm{~d}$ show these outer positions better preserved than [16]. Indeed, our distributed and asynchronous Oja's algorithm achieves in general better accuracy (around the $65 \%$ of positions) except for the third part of nodes which are located around the network's boundary, e.g. nodes 11 or $36-37$ for instance (see squared nodes in Figure 2d).

\subsection{Real data: FIT IoT-LAB platform of wireless sensor nodes}

\subsubsection{Platform description}

In order to obtain real RSSI values we make use of the FIT IoT-LAB platform deployed at Rennes (France). The 256 WSN430 open nodes 3 available at the platform are issued to the standard ZigBee IEEE 802.15.4 operating at 2.4 GHz. The

\footnotetext{
${ }^{3}$ See the technical specifications of WSN430 sensors https://github.com/iot-lab/iot-lab/wiki/Hardware_Wsn4 and CC2420 transceivers involve in our campaigns: http://www.ti.com/lit/ds/symlink/cc2420.pdf
} 
sensor nodes are located in two storage rooms of size $6 \times 15 \mathrm{~m}^{2}$ containing different objects. They are placed at the ceil which is $1.9 \mathrm{~m}$ height from the floor in a grid organization. Through of our user profile created in the FIT IoT-LAB's website, we run remotely several experiments involving the 50 selected sensor nodes within $5 \times 9 \mathrm{~m}^{2}$. All real data used in this section can be found in 4 . The environment parameters issued to the LNSM (1) are: $\sigma^{2}=28.16 \mathrm{~dB}, \mathrm{PL}_{0}=-61.71 \mathrm{~dB}$ and $\eta=2.44$. We set $q_{i j}=0.8 \forall i, j, q=0.85$ and $\gamma_{n}=\frac{0.015}{\sqrt{n}}$ for Algorithm 2

\subsubsection{Performance comparison}

We compare the same algorithms considered in Section 6.1 by setting the estimated positions obtained from each algorithm to the initialization of Algorithm 3 Table 1 shows the RMSE values before and after the refinement phase. In addition, we include the ratio of the accuracy improvement considering the RMSE values after and before applying the distributed MLE and the ratio regarding the number of positions over the total $N$ that are improved. The best performances are achieved by min-max, dwMDS and doMDS in terms of minimum RMSE value over the $N$ estimated positions. Nevertheless, the highest improvement is obtained with the proposed doMDS since the RMSE before the refinement phase was higher than the values from min-max and dwMDS which do not experiment a considerable decrease. In general, the refinement Algorithm 2 improves almost all the positions for each method and especially the anchor-free methods based on the MDS approach. Indeed, the highest values are those from the distributed versions which may exploit in advantage the local knowledge of each sensor node.

\footnotetext{
${ }^{4}$ Data base available at G. Morral personal website http://perso.telecom-paristech.fr/ morralad/
} 


\section{Conclusion}

This paper introduced a novel algorithm based on Oja's algorithm for selflocalization in wireless sensor networks. Our algorithm is based on a distributed PCA of a similarity matrix which is learned on-line. Almost sure convergence of the method is demonstrated in the context of vanishing step size. The algorithm can be coupled with a distributed maximum likelihood estimator to refine the sensors positions if needed. Numerical results have been conducted on both simulated and

real data on a WSN testbed. Although we focused on fixed sensors positions, the on-line nature of the algorithm makes it suitable for use in dynamic environments where one seek to track the position of moving sensors.

\section{Appendix A. Proof of Proposition 1}

$$
\begin{aligned}
& \text { Set for each } i, \sum_{j} \boldsymbol{M}(i, j) \boldsymbol{U}_{n-1}(j)=\left(\boldsymbol{M} \boldsymbol{U}_{n-1}\right)_{i} \text { and } \\
& \xi_{n}(i)=\left(\boldsymbol{Y}_{n}(i)-\left(\boldsymbol{M} \boldsymbol{U}_{n-1}\right)_{i}\right)+\boldsymbol{U}_{n-1}(i)\left(\boldsymbol{U}_{n-1}^{T} \boldsymbol{M} \boldsymbol{U}_{n-1}-\boldsymbol{\Lambda}_{n}(i)\right)
\end{aligned}
$$

Then, the sequence generated by each sensor node $i$ is written as:

$$
\boldsymbol{U}_{n}(i)=\boldsymbol{U}_{n-1}(i)+\gamma_{n}\left(\left(\boldsymbol{M} \boldsymbol{U}_{n-1}\right)_{i}-\boldsymbol{U}_{n-1}(i)\left(\boldsymbol{U}_{n-1}^{T} \boldsymbol{M} \boldsymbol{U}_{n-1}\right)\right)+\gamma_{n} \xi_{n}(i)
$$

Denote by $\mathcal{F}_{n}$ the $\sigma$-algebra generated by all random variables defined up to time $n$. Using Lemmas 112 and 3, it is immediate to check that $\mathbb{E}\left(\xi_{n} \mid \mathcal{F}_{n-1}\right)=0$ and thus 
the sequence $\sum_{k \leq n} \gamma_{k} \xi_{k}$ is $\mathcal{F}_{n}$-adapted martingale. We estimate

$$
\begin{aligned}
\mathbb{E}\left[\left\|\xi_{n}(i)\right\|^{2} \mid \mathcal{F}_{n-1}\right] \leq & \mathbb{E}\left[\left\|\boldsymbol{Y}_{n}(i)\right\|^{2} \mid \mathcal{F}_{n-1}\right]+\left\|\boldsymbol{U}_{n-1}(i)\right\|^{2} \mathbb{E}\left[\left\|\boldsymbol{\Lambda}_{n}(i)\right\|^{2} \mid \mathcal{F}_{n-1}\right] \\
& +2\left\|\boldsymbol{U}_{n-1}(i)\right\| \mathbb{E}\left[\left\|\boldsymbol{Y}_{n}(i) \boldsymbol{\Lambda}_{n}(i)\right\| \mid \mathcal{F}_{n-1}\right]
\end{aligned}
$$

The first term on the right hand side (RHS) of (A.2) can be expanded as:

$$
\begin{aligned}
\mathbb{E}\left[\left\|\boldsymbol{Y}_{n}(i)\right\|^{2} \mid \mathcal{F}_{n-1}\right] \leq & \mathbb{E}\left[\left|\widehat{\boldsymbol{M}}_{n}(i, i)\right|^{2}\right]\left\|\boldsymbol{U}_{n-1}(i)\right\|^{2} \\
& +\frac{N}{q} \sum_{j \neq i} \mathbb{E}\left[\left|\widehat{\boldsymbol{M}}_{n}(i, j)\right|^{2}\right]\left\|\boldsymbol{U}_{n-1}(i)\right\|^{2} \\
& +2 \sum_{j \neq i} \mathbb{E}\left[\widehat{\boldsymbol{M}}_{n}(i, i) \widehat{\boldsymbol{M}}_{n}(i, j)\right]\left\|\boldsymbol{U}_{n-1}(i)\right\|^{2}
\end{aligned}
$$

Upon noting that for any $i, j \mathbb{E}\left[\boldsymbol{S}_{n}(i, j)^{2}\right]=\frac{1}{q_{i j}} d_{i, j}^{4} C^{8}$ and $\boldsymbol{U}_{n-1}$ lies in a fixed compact set, there exists a constant $K^{\prime}$ such that $\mathbb{E}\left[\left\|\boldsymbol{Y}_{n}(i)\right\|^{2} \mid \mathcal{F}_{n-1}\right] \leq K^{\prime}$ for all $n$ depending on $N, q_{\min }=\min _{i, j} q_{i j}, C$ defined in (2) and $\max _{i, j} d_{i, j}^{4}$ such that $\mathbb{E}\left[\left|\widehat{\boldsymbol{M}}_{n}(i, j)\right|^{2}\right]<K$ for some constant $K$. The second term on the RHS of A.2 can be handled similarly:

$$
\begin{aligned}
\mathbb{E}\left[\left\|\boldsymbol{\Lambda}_{n}(i)\right\|^{2} \mid \mathcal{F}_{n-1}\right] \leq & \mathbb{E}\left[\left\|\boldsymbol{Y}_{n}(i)\right\|^{2} \mid \mathcal{F}_{n-1}\right]\left\|\boldsymbol{U}_{n-1}(i)\right\|^{2}+\left(\frac{N}{q} \sum_{j \neq i} \mathbb{E}\left[\left\|\boldsymbol{Y}_{n}(j)\right\|^{2} \mid \mathcal{F}_{n-1}\right]\right. \\
& \left.+2 \sum_{j \neq i} \mathbb{E}\left[\boldsymbol{Y}_{n}(i) \boldsymbol{Y}_{n}(j) \mid \mathcal{F}_{n-1}\right]\right)\left\|\boldsymbol{U}_{n-1}(j)\right\|^{2} \leq K^{\prime \prime}
\end{aligned}
$$


for some constant $K^{\prime \prime}$. Finally,

$$
\begin{aligned}
\mathbb{E}\left[\left\|\boldsymbol{Y}_{n}(i) \boldsymbol{\Lambda}_{n}(i)\right\| \mid \mathcal{F}_{n-1}\right] \leq & \mathbb{E}\left[\left\|\boldsymbol{Y}_{n}(i)\right\|^{2} \mid \mathcal{F}_{n-1}\right]\left\|\mathcal{F}_{n-1}(i)\right\| \\
& +\sum_{j \neq i} \mathbb{E}\left[\boldsymbol{Y}_{n}(i) \boldsymbol{Y}_{n}(j) \mid \mathcal{F}_{n-1}\right]\left\|\mathcal{F}_{n-1}(j)\right\|
\end{aligned}
$$

is uniformly bounded as well. Therefore, we have shown that a.s.

$$
\sup _{n} \mathbb{E}\left[\left\|\xi_{n}(i)\right\|^{2} \mid \mathcal{F}_{n-1}\right]<\infty
$$

Since $\sum_{n} \gamma_{n}^{2}<\infty$, it follows that $\sum_{n} \gamma_{n}^{2} \mathbb{E}\left[\left\|\xi_{n}(i)\right\|^{2} \mid \mathcal{F}_{n-1}\right]<\infty$ a.s. By Doob's Theorem, the martingale $\sum_{k \leq n} \gamma_{k} \xi_{k}(i)$ converges almost surely to some random variable finite almost everywhere. This completes the proof.

\section{Appendix B. Proof of Theorem 4}

Consider the following Lyapunov function $V: \mathbb{R}^{N \times p} \backslash\{0\} \rightarrow \mathbb{R}^{+}$:

$$
V(\boldsymbol{U})=\frac{e^{\|\boldsymbol{U}\|^{2}}}{\boldsymbol{U}^{T} \boldsymbol{M U}}
$$

The following properties hold:

i) $\lim _{\|\boldsymbol{U}\| \rightarrow \infty} V(\boldsymbol{U})=+\infty$ and the gradient is $\nabla V(\boldsymbol{U})=-2 \frac{V(\boldsymbol{U})}{\boldsymbol{U}^{T} \boldsymbol{M U}} h(\boldsymbol{U})$.

ii) $\langle V(\boldsymbol{U}), h(\boldsymbol{U})\rangle \leq 0$ and the equality holds iff $\left\{\boldsymbol{U} \in \mathbb{R}^{N \times p} \mid h(\boldsymbol{U})=0\right\}$.

The proof is an immediate consequence of Proposition 1, the existence of (B.1) along with Theorem 2 of [31]. Sequence $\boldsymbol{U}_{n}$ converges a.s. to the roots of $h$. The latter roots are characterized in [24]. In particular, $h(\boldsymbol{U})=0$ implies that each column of $\boldsymbol{U}$ is an unit-norm eigenvector of $\boldsymbol{M}$. 


\section{References}

\section{References}

[1] I. Borg, P. Groenen, Modern Multidimensional Scaling: theory and applications, New York: Springer-Verlag, 1997.

[2] Y. Shang, W. Ruml, M. Fromherz, Localization from mere connectivity, in: Proceedings of the 4th ACM International Symposium on Mobile Ad Hoc Networking \&Amp; Computing, MobiHoc '03, ACM, 2003, pp. 201-212.

[3] T. Rappaport, Wireless Communications: Principles and Practice, Prentice Hall, 1996.

[4] N. Patwari, et al., Locating the Nodes : cooperative localization in wireless sensor networks, IEEE Signal Processing Magazine 22 (4) (2005) 54-69.

[5] G. Mao, B. Fidan, B. Anderson, Wireless sensor network localization techniques, Computer Networks 51 (10) (2007) 2529-2553.

[6] W. Hereman, Trilateration: The Mathematics Behind a Local Positioning System, seminar (June 2011).

[7] A. Savvides, H. Park, M. Srivastava, The Bits and Flops of the N-hop Multilateration Primitive For Node Localization Problems, in: Proceedings of the 1st ACM International Workshop on Wireless Sensor Networks and Applications, WSNA '02, ACM, 2002, pp. 112-121.

[8] D. Niculescu, B. Nath, Ad Hoc Positioning System (APS), in: IN GLOBECOM, 2001, pp. 2926-2931. 
[9] C. Savarese, J. Rabaey, K. Langendoen, Robust positioning algorithm for distributed ad-hoc wireless sensor network, in: Proceedings of the General Track of the Annual Conference on USENIX Annual Technical Conference, Monterey, 2002, pp. 317-327.

[10] N. Patwari, R. J'Odea, W. Yanwei, Relative location in wireless networks, in: VTC, 2001.

[11] K. Kaemarungsi, P. Krishnamurthy, Modeling of Indoor Positioning Systems Based on Location Fingerprinting, in: INFOCOM, 2004.

[12] J. Xu, W. Liu, F. Lang, Y. Zhang, C. Wang, Distance measurement model based on rssi in wsn., Wireless Sensor Network 2 (8) (2010) 606-611.

[13] N. Dieng, M. Charbit, C. Chaudet, L. Toutain, T. Meriem, Indoor Localization in Wireless Networks based on a Two-modes Gaussian Mixture Model, in: IEEE 78th Vehicular Technology Conference (VTC Fall), 2013, pp. 1-5.

[14] J. Leeuw, Applications of Convex Analysis to Multidimensional Scaling., Recent Developments in Statistics (1977) 133-145.

[15] P. Biswas, T. Liang, T. Wang, Y. Ye, Semidefinite programming based algorithms for sensor network localization, ACM Transactions on Sensor Networks 2.

[16] J. Costa, N. Patwari, A. Hero, Distributed Weighted-Multidimensional Scaling for Node Localization in Sensor Networks, ACM Transactions on Sensor Networks 2 (1) (2006) 39-64. 
[17] M. Essoloh, C. Richard, H. Snoussi, Localisation distribuée dans les réseaux de capteurs sans fil par résolution d'un problème quadratique, in: GRETSI, 2007.

[18] P. Biswas, Y. Ye, A Distributed Method for Solving Semidefinite Programs Arising from Ad Hoc Wireless Sensor Network Localization, in: Multiscale Optimization Methods and Applications, Vol. 82 of Nonconvex Optimization and Its Applications, Springer US, 2006, pp. 69-84.

[19] F. Cattivelli, A. Sayed, Distributed nonlinear Kalman filtering with applications to wireless localization, in: Acoustics Speech and Signal Processing (ICASSP), 2010 IEEE International Conference on, Dallas, TX, 2010, pp. $3522-3525$.

[20] N. Trawny, S. Roumeliotis, G. Giannakis, Cooperative multi-robot localization under communication constraints, in: Robotics and Automation, 2009. ICRA '09. IEEE International Conference on, Kobe, 2009, pp. 4394 - 4400.

[21] Y. Shang, W. Ruml, Improved MDS-based localization, in: INFOCOM 2004. Twenty-third AnnualJoint Conference of the IEEE Computer and Communications Societes, Hong Kong, 2004, pp. 2640 - 2651 vol.4.

[22] S. Korada, A. Montanari, S. Oh, Gossip pca, ACM SIGMETRICS Performance Evaluation Review 39 (1) (2011) 169-180.

[23] G. Morral, P. Bianchi, J. Jakubowicz, Asynchronous Distributed Principal Component Analysis Using Stochastic Approximation, in: Decision and Control (CDC), 2012 IEEE 51st Annual Conference on, Maui, Hawaii, 2012, pp. $1398-1403$. 
[24] E. Oja, Principal components, minor components, and linear neural networks, Journal of Neural Networks 5 (6) (1992) 927-935.

[25] V. Borkar, S. Meyn, Oja's algorithm for graph clustering, markov spectral decomposition, and risk sensitive control, Journal of Automatica 48 (10) (2012) 2512-2519.

[26] V. Borkar, Stochastic approximation: a dynamical system viewpoint, Cambridge University Press, 2008.

[27] J. Tsitsiklis, Problems in Decentralized Decision Making and Computation, Ph.D. thesis, Massachusetts Institute of Technology (1984).

[28] P. Bianchi, G. Fort, W. Hachem, Performance of a Distributed Stochastic Approximation Algorithm, IEEE Transactions on Information Theory 59 (11) (2013) $7405-7418$.

[29] G. Morral, P. Bianchi, G. Fort, Success and Failure of AdaptationDiffusion Algorithms for Consensus in Multi-Agent Networks, Arxiv preprint arXiv:1410.6956.

[30] A. Nedic, A. Olshevsky, Stochastic Gradient-Push for Strongly Convex Functions on Time-Varying Directed Graphs, Arxiv preprint arXiv:1406.2075.

[31] B. Delyon, Stochastic Approximation with Decreasing Gain: Convergence and Asymptotic Theory, Unpublished Lecture Notes, http://perso.univrennes1.fr/bernard.delyon/as_cours.ps. 


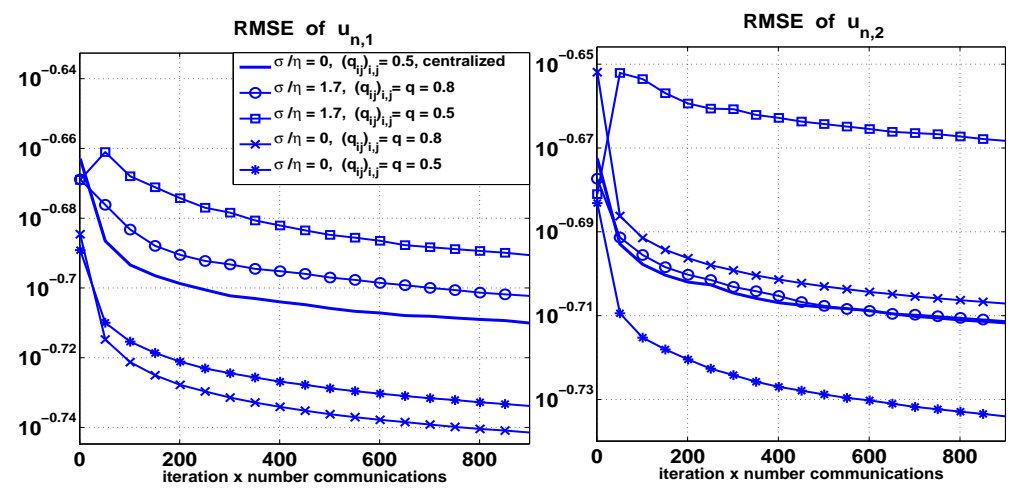

Figure 1: RMSE as a function of $n N$ from the two estimated eigenvectors $\boldsymbol{u}_{n, 1}$ and $\boldsymbol{u}_{n, 2}$ when considering the noiseless and noisy case and for different values of $q$.

\begin{tabular}{|l|c|c|c|c|c|c|}
\hline Method & MC & min-max & MDS & Oja & dwMDS & doMDS \\
\hline Before refinement & 1.87 & 0.8 & 1.98 & 2.18 & 0.86 & 1.56 \\
\hline After refinement & 1.05 & 0.54 & 1.39 & 1.37 & 0.6 & 0.51 \\
\hline Improvement (\%) & 44 & 32 & 30 & 28 & 30 & 78 \\
\hline Positions improved (\%) & 75 & 71 & 80 & 80 & 82 & 86 \\
\hline
\end{tabular}

Table 1: RMSE averaged over the 44 estimated positions considering real data. 


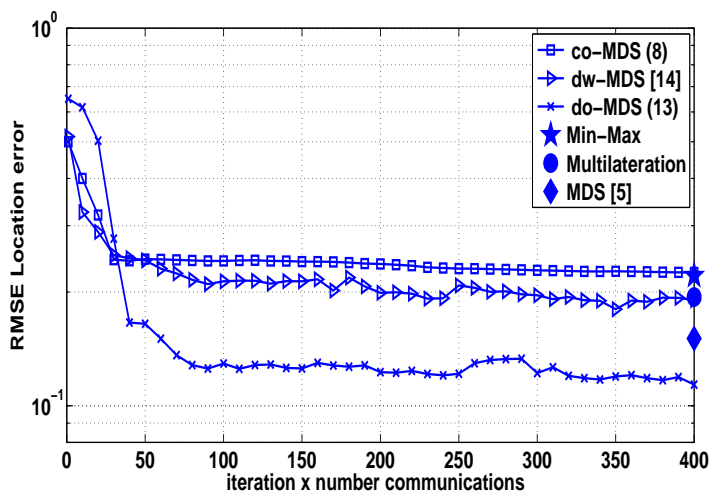

(a) RMSE as a function of $n N$ from the estimated positions $\left(\widehat{\boldsymbol{Z}}_{n}(1), \ldots, \widehat{\boldsymbol{Z}}_{n}(N)\right)$.
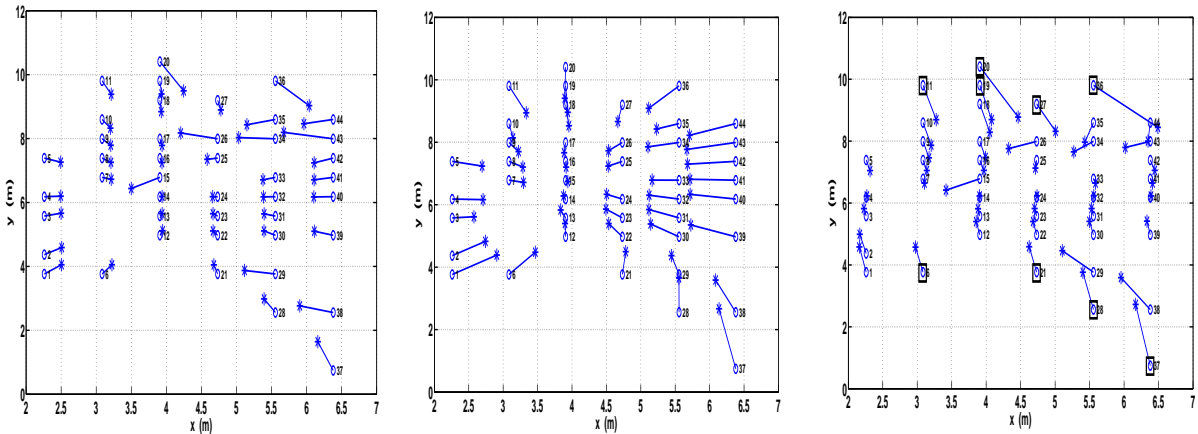

(b) Oja's algorithm (6)-(7).

(c) dwMDS [16].

(d) Algorithm 2(doMDS).

Figure 2: Estimated positions after 1000 iterations. Markers (*) correspond to the estimated values while markers $(\bigcirc)$ to the true positions. Squared positions $(\square)$ in d) highlight worse accuracy compared to b). 